\section{British Bees and Wind-borne Pollen}

IT is well known that the perianth in windpollinated plants is lacking or greatly reduced and inconspicuous, that nectar-secreting glands are absent and that immense quantities of pollen are produced compared with entomophilous flowers. Furthermore, the individual pollen grains are more suited to carriage by wind, with their thinner walls and simpler form lacking spines or surface sculpture. They have a markedly lower specific gravity and do not cohere en masse like those of insect-fertilized flowers. It is therefore rather surprising to find that solitary bees of the genus Andrena sometimes collect large quantities of anemophilous pollen, for example, Quercus, Fagus, Castanea, as the analyses per cent of individual pollen loads taken off the species named below demonstrate :

\begin{tabular}{lrrrlr}
\multicolumn{5}{c}{ A. hamorrhoa (Fab.). } \\
Quercus & 83 & Fagus 2 & $82 \cdot 2$ & Fagus & $88 \cdot 7$ \\
Ranunculus & 8 & Taraxacum & $13 \cdot 0$ & Bellis & $7 \cdot 7$ \\
Crataegus & 6 & Cruciferæ & $3 \cdot 8$ & Others & $3 \cdot 6$ \\
Acer & 3 & Others & $1 \cdot 0$ & &
\end{tabular}

\begin{tabular}{crlllllr}
\multicolumn{8}{c}{ A. armata (Gmelin) } \\
4 & \multicolumn{9}{c}{6} & 7 \\
Quercus & $90 \cdot 6$ & Quercus & $56 \cdot 3$ & Quercus & $56 \cdot 7$ & Quercus & $97 \cdot 1$ \\
Viburnam & $9 \cdot 4$ & Acer & $28 \cdot 6$ & Acer & $41 \cdot 3$ & Ilex & $2 \cdot 9$ \\
& & Ilex & $13 \cdot 2$ & Ilex & $2 \cdot 0$ & &
\end{tabular}

\begin{tabular}{lrlr}
\multicolumn{5}{c}{ A. pubescens Oliv. } \\
Fagus 8 & $51 \cdot 1$ & Fagus & \\
Taraxacum & $21 \cdot 1$ & Acer & $26 \cdot 8$ \\
Heracleum & $14 \cdot 3$ & Taraxacum & $17 \cdot 4$ \\
Prunus & $11 \cdot 7$ & Salix & $1 \cdot 6$ \\
Others & $1 \cdot 8$ & Others & $0 \cdot 6$
\end{tabular}

\begin{tabular}{lrl|rllll}
\multicolumn{8}{c}{ A. bimaculata (Kirby) (2nd brood). } \\
Castanea & 99 & Castanea & $61 \cdot 4$ & Castanea & $78 \cdot 1$ & Castanea & $36 \cdot 2$ \\
Spircea & 1 & Spirca & $34 \cdot 4$ & Rubus & $21 \cdot 9$ & Spircea & $63 \cdot 8$ \\
& & Rubus & $4 \cdot 2$ & & & &
\end{tabular}

In addition, pollen loads containing high proportions of Quercus pollen have been taken off $A$. jacobi Perk., and of Castanea pollen off $A$. dorsata (Kirby) and A. thoracica (Fab.).

The factors governing the choice of flowers visited by bees are probably numerous; even the extensive investigations on the hive bee (Apis mellifera L.) have led to few definite conclusions ${ }^{1}$. Consideration of the results tabulated above raises several interesting queries.

In the first place, dealing with examples (7) and (10): how do the bees manage to carry a practically pure load of poorly cohering Quercus or Castanea pollen diluted with only a small proportion of more 'sticky' pollen from nectariferous flowers? In the hive bee, where separate journeys for nectar or pollen are normal, the pollen-carriers moisten the pollen with nectar to assist carriage, thus involving an appreciable number of visits to nectariferous blooms. It is difficult to imagine this being done without taking pollen from these flowers as well.

Secondly, what factors are responsible for diverting the bees from their normal pollen sources for tall trees with inconspicuous nectarless flowers, which, in the case of Fagus and Quercus, are very much obscured by foliage? The remaining pollen species in the loads analysed are derived from low herbs or shrubs which have conspicuous flowers or are rich sources of nectar or both, and all were in sufficient abundance in the neighbourhood of the colonies from which the bees were taken upon their return. The taking of anemophilous pollen is not normal in
A. hoemorrhoa, A. armata and A. pubescens, as only 4, 5 and 3 loads containing appreciable quantities have been found among totals of 42,33 and 36 loads analysed respectively. The catkins of Castanea are much more conspicuous than those of Fagus and Quercus, which may partly explain the frequent occurrenee of this pollen species as a major constituent of loads of $A$. bimaculata taken in localities where this tree abounds. It should be noted, however, that Castanea has previously been classified as entomophilous ${ }^{2}$.

A possible explanation for the taking of Fagus and Quercus pollen by Andrena may be as follows. When the tree is in full flower and immense quantities of pollen are being liberated, it is reasonable to suppose that an atmospheric pollen concentration gradient will be set up around the tree, with a direction and rate of decrease in concentration depending upon the wind. A bee flying into this 'pollen field' will make a chemotropic response to the floating grains, and will orientate itself in the general direction of increasing pollen concentration and travel up the gradient until it arrives at the pollen source. Where the concentration of atmospheric pollen is low or uniform, the bee will make no directional response.

Although this suggestion can only be regarded as speculation until evidence based upon carefully chosen series of observations is available, the following experience may have some bearing upon it. A colony of $A$. barbilabris (Kirby) situated in a large plantation of Scots pine (Pinus sylvestris), in flower during the flight period of this species, was studied in 1943 and 1944. In the 83 pollen loads examined, Pinus was found only in negligible traces, the bees collecting pollen from nectariferous plants several hundred yards distant from the colony. Setting aside the possibility that Pinus pollen may be distasteful, upon the hypothesis put forward, the presence of a high but uniform atmospheric concentration of this pollen from the dense mass of trees had no orientating effect upon the bees' flight.

I hope to publish in due course detailed analyses of pollen loads of Andrena.

47 Westbourne Road,

V. H. Chambers. Luton.

${ }^{1}$ Weiss, H. B., J. Econ. Ent., 36, 1 (1943), a review.

${ }^{2}$ Hyde, H. A., and Williams, D. A., Nex Phyt., 43, 49 (1944).

\section{Transmission of Kala-Azar to the Pouch Young of the Common Australian Possum (Trichosurus vulpecula)}

KALA-AZAR is difficult to transmit to laboratory animals, with the exception of the hamster (Cricetulus griseus). This rodent, however, is not available in many countries.

Recently, through the courtesy of Dr. G. A. H. Heydon, School of Tropical Medicine, Sydney, a culture of kala-azar on N.N.N. medium was obtained, which was injected intraperitoneally into a pouch young of Trichosurus vulpecula about $2 \frac{1}{2}$ months old. The young, which at the time was practically hairless and still firmly attached to the nipple, was lifted out of the pouch of the anæsthetized mother, injected with $1 \mathrm{ml}$. of the broth washings of the culture tube and replaced into the pouch. One month after the injection, the young, still only covered by hairs 